\begin{tabular}{|l|}
\hline Edited by Kiriakos Xenitidis and Colin Campbell \\
\hline Contents \\
- Letter to the Editor about 'Mental health difficulties \\
across childhood and mental health service use: \\
findings from a longitudinal population-based \\
study' \\
- Author's reply
\end{tabular}

\section{Letter to the Editor about 'Mental health difficulties across childhood and mental health service use: find- ings from a longitudinal population-based study'}

We read with great interest the article published by Mulraney et $\mathrm{al}^{1}$ in the July 2020 issue. This was an extensive study and targeted a very vulnerable group of our society. The study was followed up longitudinally and pointed to the deficits in utilisation of mental health services. Although this study provided great insights, we had some reservations that we would like to discuss.

The authors have clearly described the take up of the data from the Longitudinal Study of Australian Children cohort. Clearly defined inclusion and exclusion criteria with a description of the recruitment process and how contact was made or how the questionnaire was applied, would have made the study design easier to follow. The recruitment process was difficult to infer especially for non-English-speaking readers.

The authors mentioned that children excluded from the analysis were likely to have less educated parents, more mental health problems and disturbed family dynamics but we think that these are one of the most vulnerable groups, who need a higher level of care. $^{2}$ The risk of underrepresentation of these subpopulations probably skewed the data, as those with higher unmet needs were left out of the study. Also, lack of mention of statistically significant values for the Vuong-Lo-Mendell-Rubin likelihood ratio test made it a little hard to interpret the results.

The authors have very rightly projected four trajectories - low symptoms, high decreasing, moderate increasing and high increasing - but while following the tables in the supplementary data with the trajectories and individual specialist contact or in different waves, it was difficult to follow through. The authors have reported an attrition of 3764 participants, which was not reflected in these tables. Also, the reason for drop-out of participants from 4348 at end of the wave 1 to 3764 at the end of wave 6 would have made it more informative. Other factors like dual or referred consultation, changes in outcome, ease of accessibility and presence of required services in the defined postcode could play a role in the proposed conclusions. ${ }^{3}$ Probably the ratio of children who benefited with or without an adequate number of visits could have provided insights into the study.

What actually is of real concern is that psychiatrists and psychologists are the least consulted ones for mental health issues. A possible role of stigmatised views, of parents and society, towards mental health cannot be overlooked.

There are minor typographical errors that we noticed that were misleading like 'Supplementary Fig. 1 shows' in the results, while no such figure has been provided in the supplementary material or in Figure 1 of the article; age has been defined from 4 to 14 years, but in the graph that follows, age ' 10 ' has been followed by ' 15 ' and then by ' 14 '.

It would be a great help if the authors could clarify these points. Thank you.

\section{Declaration of interest}

None.

\section{References}

1 Mulraney M, Hiscock H, Sciberras E, Coghill D, Sawyer M. Mental health difficulties across childhood and mental health service use: findings from a longitudinal population-based study. Br J Psychiatry 2020; 217: 364-9.

2 Lyon AR, Ludwig KA, Stoep AV, Gudmundsen G, McCauley E. Patterns and predictors of mental healthcare utilization in schools and other service sectors among adolescents at risk for depression. School Ment Health 2013; http//doi. org/10.1007/s12310-012-9097-6.

3 Reid GJ, Stewart SL, Barwick M, Carter J, Leschied A, Neufeld RWJ, et al. Predicting patterns of service utilization within children's mental health agencies. BMC Health Serv Res 2019; 19: 993.

Isha Batra; Avni Gupta, Department of Psychiatry, Pt. B. D. Sharma PGIMS Rohtak, India; Kajal Taneja, Department of Psychiatry, Pt. B. D. Sharma PGIMS Rohtak, India; Aparna Goyal, Department of Psychiatry, Pt. B. D. Sharma PGIMS Rohtak, India. Email: isha.batra45@gmail.com

doi:10.1192/bjp.2021.77

\section{Author's reply}

We thank Batra and colleagues for their comments about our article and respond to their queries below.

The design of the Longitudinal Study of Australian Children has been thoroughly described previously, ${ }^{1}$ we provide a brief overview in our article but did not have the space to go into detail. In regards to the areas of particular concern raised: all children enrolled in the Australian Medicare system who were aged 4 years or $<12$ months during recruitment were eligible to participate; the only exclusion was that only one child from each family could participate, thus in the instance of multiple births only one child from the family was eligible. The data were collected using a combination of surveys and interviews. In instances where parents had insufficient English reading ability to complete the survey then an interview was conducted and for those of non-English speaking background interpreters were available.

We agree that the exclusion of those with less educated parents, more mental health problems and disturbed family dynamics from analyses may have skewed the results. However, our primary finding was that children are not having their mental health needs met under the current healthcare system. Thus, having data to analyse from these more vulnerable groups would likely not have had an impact on our conclusions.

We used Mplus to conduct our latent class growth analysis, using full information maximum likelihood (FIML) to account for missing data. To ensure our findings were rigorous and reflected the actual data as closely as possible we chose to only include participants with data from at least three time points in analyses. The Medicare data we used for health service usage is collected through administrative data linkage and as such there is not an impact from attrition. Although there was sample attrition in terms of surveys completed over the six waves of data collection, few families withdrew from the study. Thus, our use of FIML and data linkage resulted in our analytic sample of $n=4348$.

Batra \& Sharma raise an excellent point about whether children who receive services benefitted from these. We have since completed further analyses that show that the receipt of intervention does not appear to improve outcomes for these children. ${ }^{2}$

Thank you for pointing out that the supplementary figure was missing, we have sent this to the Journal and requested they add it to the supplementary material. Figure 1 in the article contains a typo, the number ' 15 ' on the $x$-axis should be the number ' 12 '. 